\title{
STRONG STATISTICAL STABILITY OF NON-UNIFORMLY EXPANDING MAPS
}

\author{
JOSÉ F. ALVES
}

\begin{abstract}
We consider families of transformations in multidimensional Riemannian manifolds with non-uniformly expanding behavior. We give sufficient conditions for the continuous variation (in the $L^{1}$-norm) of the densities of absolutely continuous (with respect to the Lebesgue measure) invariant probability measures for those transformations.
\end{abstract}

\section{INTRODUCTION}

In this work we address ourselves to the study of the statistical stability of certain classes of chaotic dynamical systems. We are particularly interested in the statistical stability of systems displaying non-uniformly expanding behavior on the growth of the derivative for most of its orbits.

To be more specific, let $f: M \rightarrow M$ be some discrete-time dynamical system of a compact Riemannian manifold $M$, and let $m$ be a volume form that we call Lebesgue measure. Sinai-Ruelle-Bowen (SRB) measures or physical measures are probability measures that characterize asymptotically, in time average, a large set of orbits of the phase space; these are defined precisely in (3) below. It is a difficult problem to verify the existence of these measures for general dynamical systems.

By the statistical stability of a system, we mean continuous variation of the SRB measures under small modifications of the law that governs the system. Using Birkhoff's Ergodic Theorem, one possible way for finding SRB measures for a map $f$ is by proving the existence of ergodic absolutely continuous $f$-invariant probability measures.

Systems displaying uniformly expanding behavior have been exhaustively studied in the last decades, and several results on the existence of SRB measures and their statistical stability have been obtained, starting with Sinai, Ruelle and Bowen; see 16, 14, 9, 10] and also [15, 12, 13, 18.

The existence of SRB measures for many one-dimensional maps with non-uniformly expanding behavior has been established in the pioneer work of Jakobson [1]; see also [7, 8, 6]. Viana introduced in [17] an open class of transformations in higher dimensions with non-uniformly expanding behavior for most of its orbits. The existence of SRB measures for Viana maps has been proved in [1]. Motivated by the results in [17] and 1], general conclusions on the existence of SRB measures for non-uniformly expanding dynamical systems are drawn in 3 .

The statistical stability of the systems introduced in [17] has been proved in [5], in a strong sense: convergence of the densities of the SRB measures in the $L^{1}$ norm. The proof uses in an important way geometrical features of the system, and could not be immediately extended to more general classes of non-uniformly expanding maps. Some

Date: November 13, 2018.

1991 Mathematics Subject Classification. 37C40, 37C75, 37D25.

Key words and phrases. Non-uniformly expanding maps, SRB measures, statistical stability.

Work partially supported by FCT through CMUP. 
results in this direction were obtained in [2], but in a weak sense: convergence of the measures in the weak* topology.

In this work we give sufficient conditions for the strong statistical stability of certain classes of non-uniformly expanding maps. These conditions are naturally verified by the maps introduced in [17, as shown in [5], and by a class of non-uniformly expanding local diffeomorphisms introduced in [3] that we include at the end of this work.

1.1. Non-uniformly expanding maps. Let $f: M \rightarrow M$ be a continuous map which is local diffeomorphism in the whole manifold except in a set of critical points $\mathcal{C} \subset M$.

Definition 1.1. We say that $\mathcal{C}$ is non-degenerate if the following conditions hold. The first one says that $f$ behaves like a power of the distance to $\mathcal{C}$ : there are $B>1$ and $\beta>0$ such that for every $x \in M \backslash \mathcal{C}$

$\left(\mathrm{s}_{1}\right) \quad B^{-1} \operatorname{dist}(x, \mathcal{C})^{\beta} \leq\|D f(x) v\| \leq B \operatorname{dist}(x, \mathcal{C})^{-\beta}$, for all $v \in T_{x} M$ with $\|v\|=1$. Moreover, we assume that $\log |\operatorname{det} D f|$ and $\log \left\|D f^{-1}\right\|$ are locally Lipschitz in $M \backslash \mathcal{C}$, with Lipschitz constant depending on the distance to $\mathcal{C}$ : for every $x, y \in M \backslash \mathcal{C}$ with $\operatorname{dist}(x, y)<\operatorname{dist}(x, \mathcal{C}) / 2$ we have

$$
\begin{array}{ll}
\left(\mathrm{s}_{2}\right) \quad & |\log |\left|D f(x)^{-1}\|-\log \| D f(y)^{-1} \|\right| \leq \frac{B}{\operatorname{dist}(x, \mathcal{C})^{\beta}} \operatorname{dist}(x, y) ; \\
\left(\mathrm{s}_{3}\right) \quad & |\log | \operatorname{det} D f(x)|-\log | \operatorname{det} D f(y)|| \leq \frac{B}{\operatorname{dist}(x, \mathcal{C})^{\beta}} \operatorname{dist}(x, y) .
\end{array}
$$

Given $\delta>0$ and $x \in M \backslash \mathcal{C}$ we define the $\delta$-truncated distance $\operatorname{dist}_{\delta}(x, \mathcal{C})=\operatorname{dist}(x, \mathcal{C})$, if $\operatorname{dist}(x, \mathcal{C})<\delta$, and $\operatorname{dist}_{\delta}(x, \mathcal{C})=1$, otherwise.

Definition 1.2. Let $f: M \rightarrow M$ be a local diffeomorphism outside a non-degenerate critical set $\mathcal{C}$. We say that $f$ is non-uniformly expanding if:

- there is $\lambda>0$ such that for every $x \in M$

$$
\limsup _{n \rightarrow \infty} \frac{1}{n} \sum_{i=0}^{n-1} \log \left\|D f\left(f^{i}(x)\right)^{-1}\right\|<-\lambda
$$

- for every $\epsilon>0$ there exists $\delta>0$ such that for every $x \in M$

$$
\limsup _{n \rightarrow+\infty} \frac{1}{n} \sum_{j=0}^{n-1}-\log \operatorname{dist}_{\delta}\left(f^{j}(x), \mathcal{C}\right) \leq \epsilon .
$$

We will often refer to (2) by saying that orbits have slow recurrence to the critical set $\mathcal{C}$. When $\mathcal{C}=\emptyset$ we simply ignore the slow recurrence condition.

Remark 1.3. Slow recurrence condition is not needed in all its strength. In fact, the only place where we will be using (2) is in the proof of Proposition 3.5. As we shall see, it is enough that (2) holds for some sufficiently small $\epsilon>0$ and conveniently chosen $\delta>0$; see Remark 3.6.

A Borel probability measure $\mu$ on the Borel sets of $M$ is said to be an SRB measure if there exists a positive Lebesgue measure set of points $z \in M$ for which

$$
\lim _{n \rightarrow+\infty} \frac{1}{n} \sum_{j=0}^{n-1} \varphi\left(f^{j}(z)\right)=\int \varphi d \mu
$$

for any continuous function $\varphi: M \rightarrow \mathbb{R}$. The set of points $z \in M$ for which this holds is called the basin of $\mu$. It was proved in [3] that non-uniformly expanding maps possess SRB measures. 
If $f: M \rightarrow M$ is non-uniformly expanding, then by (11) the expansion time function

$$
\mathcal{E}(x)=\min \left\{N \geq 1: \frac{1}{n} \sum_{i=0}^{n-1} \log \left\|D f\left(f^{i}(x)\right)^{-1}\right\| \leq-\lambda, \quad \text { for all } n \geq N\right\}
$$

is defined and finite almost everywhere in $M$. Then, according to Remark 1.3, we fix $\varepsilon>0$ and $\delta>0$ as in (2). The recurrence time function

$$
\mathcal{R}(x)=\min \left\{N \geq 1: \frac{1}{n} \sum_{i=0}^{n-1}-\log \operatorname{dist}_{\delta}\left(f^{j}(x), \mathcal{C}\right) \leq \varepsilon, \quad \text { for all } n \geq N\right\}
$$

is also defined and finite almost everywhere in $M$. We define the tail set

$$
\Gamma_{n}=\{x: \mathcal{E}(x)>n \text { or } \mathcal{R}(x)>n\} .
$$

This is the set of points which at time $n$ have not yet achieved either the uniform exponential growth of derivative or the uniform slow recurrence. If $\mathcal{C}=\emptyset$, we ignore the recurrence time function in the definition of $\Gamma_{n}$.

1.2. Statistical stability. Let $\mathcal{F}$ be a family of $C^{k}$ maps $(k \geq 2)$ from a $d$-dimensional manifold $M$ into itself, and endow $\mathcal{F}$ with the $C^{k}$ topology. We assume that each $f \in \mathcal{F}$ admits a unique absolutely continuous $f$-invariant probability measure $\mu_{f}$ in $M$.

Definition 1.4. We say that $f_{0} \in \mathcal{F}$ is (strongly) statistically stable, if $\mathcal{F} \ni f \mapsto d \mu_{f} / d m$ is continuous at $f_{0}$, with respect to the $L^{1}$-norm on the space of densities.

We assume that the maps in a neighborhood of $f_{0}$ satisfy the following non-degeneracy condition: given any $\epsilon>0$ there exists $\delta>0$ such that

$$
m(E) \leq \delta \quad \Rightarrow \quad m\left(f^{-1}(E)\right) \leq \epsilon
$$

for any measurable subset $E \subset M$ and any $f \in \mathcal{F}$. This can often be enforced by requiring some jet of order $l \leq k$ of $f_{0}$ to be everywhere non-degenerate. This is obviously satisfied whenever we consider local diffeomorphisms.

Definition 1.5. We say that $\mathcal{F}$ as above is a uniform family if the $B, \beta$ as in Definition 1.1 . and $\varepsilon, \delta, \lambda$ as in Definition 1.2 (cf. Remark [3.6) can be chosen uniformly in $\mathcal{F}$.

Theorem A. Let $\mathcal{F}$ be a uniform family of $C^{k}(k \geq 2)$ non-uniformly maps for which non-degeneracy condition (71) holds. Assume that there are $C>0$ and $\gamma>1$ such that $m\left(\Gamma_{n}^{f}\right) \leq C n^{-\gamma}$, for all $n \geq 1$ and $f \in \mathcal{F}$. Then every $f \in \mathcal{F}$ is statistically stable.

Condition (7) is needed just because we are going to use [5, Theorem A]; see Theorem 2.2 below.

\section{Piecewise EXPANDING INDUCED MAPS}

One possible way for proving the existence of invariant measures for certain dynamical systems may be by choosing conveniently some region in the phase space and studying an induced return map to that region. This method can also be efficient in proving the

absolute continuity of those measures. In this section we are particulary interested in the study of those return maps. 
2.1. Markovian return maps. Let $f$ be a map from a Riemannian manifold $M$ into itself, and let $F: \Delta \rightarrow \Delta$ be a return map for $f$ in some topological disk in $\Delta \subset M$. This means that there is a countable partition $\mathcal{P}$ of a full Lebesgue measure subset of $\Delta$, and there exists a return time function $R: \mathcal{P} \rightarrow \mathbb{Z}^{+}$such that $\left.F\right|_{U}=\left.f^{R(U)}\right|_{U}$ for each $U \in \mathcal{P}$.

Definition 2.1. We say that $F$ is a piecewise expanding Markovian map if there is a countable partition $\mathcal{P}$ into open sets of a full Lebesgue measure subset of $\Delta$ such that:

(1) Expansion: there is $0<\kappa<1$ such that for each $U \in \mathcal{P}$ and $x \in U$

$$
\left\|D F(x)^{-1}\right\|<\kappa \text {. }
$$

(2) Bounded distortion: there is $K>0$ such that for each $U \in \mathcal{P}$ and $x, y \in U$

$$
\log \left|\frac{\operatorname{det} D F(x)}{\operatorname{det} D F(y)}\right| \leq K \operatorname{dist}(F(x), F(y)) .
$$

(3) Markov: $\left.F\right|_{U}$ is a $C^{2}$ diffeomorphism onto $\Delta$, for each $U \in \mathcal{P}$.

If $F: \Delta \rightarrow \Delta$ is a $C^{2}$ piecewise expanding Markovian map, then it has some absolutely continuous invariant measure $\mu_{F}$. Moreover, the density of $\mu_{F}$ is uniformly bounded by some constant; see e.g. [19, Theorem 1]. Defining

$$
\mu_{f}^{*}=\sum_{j=0}^{\infty} f_{*}^{j}\left(\mu_{F} \mid\{R>j\}\right),
$$

it is straightforward to check that $\mu_{f}^{*}$ is an absolutely continuous $f$-invariant measure, which is finite whenever $R \in L^{1}(\Delta)$.

2.2. Statistical stability. Let $\mathcal{F}$ be a family of $C^{k}$ maps $(k \geq 2)$ from the manifold $M$ into itself, and assume that we may associate to each $f \in \mathcal{F}$ a piecewise expanding return map $F_{f}: \Delta \rightarrow \Delta$ as in Definition [2.1. For each $f \in \mathcal{F}$, let $\mathcal{P}_{f}$ denote the partition into domains of smoothness of $F_{f}$ and $R_{f}: \mathcal{P}_{f} \rightarrow \mathbb{Z}^{+}$be the corresponding return time. We assume that $R_{f} \in L^{1}(\Delta)$ for each $f \in \mathcal{F}$, which then implies that if $\mu_{F}$ is the absolutely continuous $F_{f}$-invariant probability measure, then $\mu_{f}^{*}=\sum_{j=0}^{\infty} f_{*}^{j}\left(\mu_{F} \mid\left\{R_{f}>j\right\}\right)$ is an absolutely continuous $f$-invariant finite measure. In our setting of Markovian maps, the statement of [5, Theorem A] can be simplified.

Theorem 2.2. Let $\mathcal{F}$ be as above, and suppose that every $f \in \mathcal{F}$ admits a unique absolutely continuous invariant probability measure $\mu_{f}$. Suppose that each $f_{0} \in \mathcal{F}$ satisfies:

$\left(\mathrm{u}_{1}\right)$ Given $\epsilon>0$ there is $\delta>0$ such that for any $f \in \mathcal{F}$

$$
\left\|f-f_{0}\right\|_{C^{k}}<\delta \Rightarrow\left\|R_{f}-R_{f_{0}}\right\|_{1}<\epsilon .
$$

$\left(\mathrm{u}_{2}\right) \kappa, K$ as in Definition 2.1 may be taken uniformly in a neighborhood of $f_{0}$ in $\mathcal{F}$. Then $f_{0}$ is statistically stable.

Remark 2.3. The bounded distortion condition used in [5, Theorem A] is satisfied in our context, as we shall see in Lemma 4.6. Moreover, the assumption on the constants $\beta$ and $\rho$ as in condition (U3) of [5] is trivially satisfied. In the non-Markovian case treated in [5. Theorem A], one can only assure that the density of $\mu_{F}$ belongs to $L^{p}(\Delta)$ for some $p>1$. This implies that convergence of $R_{f}$ to $R_{f_{0}}$ has to be taken in the norm of $L^{q}(\Delta)$ with $p^{-1}+q^{-1}=1$. Since in our case the density belongs to $L^{\infty}(\Delta)$ we may take the convergence of $R_{f}$ to $R_{f_{0}}$ in the $L^{1}$-norm, by a usual Hölder inequality argument. 
Under the assumptions of the Theorem 2.2, the unique absolutely continuous invariant probability measure is necessarily equal to the normalization of $\mu_{f}^{*}$, i.e. $\mu_{f}=\mu_{f}^{*} / \mu_{f}^{*}(M)$. Thus for proving Theorem $\mathrm{A}$ we just have to show that conditions $\left(\mathrm{u}_{1}\right)$ and $\left(\mathrm{u}_{2}\right)$ hold for families $\mathcal{F}$ as in Theorem $\mathrm{A}$.

\section{Hyperbolic times And BOUnded Distortion}

In this section we present some results on the existence of hyperbolic times for nonuniformly expanding maps and distortion properties at hyperbolic times. Although these results have essentially been all proved in [3], we include some proofs here in order to see how the constants depend on one another.

Definition 3.1. Fix $B>1$ and $\beta>0$ as in Definition 1.1, and take $b>0$ such that $2 b<\min \left\{1, \beta^{-1}\right\}$. Given $\sigma<1$ and $\delta>0$, we say that $n$ is a $(\sigma, \delta)$-hyperbolic time for a point $x \in M$ if for all $1 \leq k \leq n$,

$$
\prod_{j=n-k}^{n-1}\left\|D f\left(f^{j}(x)\right)^{-1}\right\| \leq \sigma^{k} \quad \text { and } \quad \operatorname{dist}_{\delta}\left(f^{n-k}(x), \mathcal{C}\right) \geq \sigma^{b k} .
$$

In the case $\mathcal{C}=\emptyset$ the definition of $(\sigma, \delta)$-hyperbolic time reduces to the first condition in (9) and we simply call it a $\sigma$-hyperbolic time.

Lemma 3.2. Given $\delta>0$ fix $\delta_{1}=\delta_{1}(B, \beta, \sigma, \delta)>0$ so that $4 \delta_{1}<\delta$ and $4 B \delta_{1}<\delta^{\beta}|\log \sigma|$. If $n$ is a $(\sigma, \delta)$-hyperbolic time for $x$, then $\left\|D f(y)^{-1}\right\| \leq \sigma^{-1 / 2}\left\|D f\left(f^{n-j}(x)\right)^{-1}\right\|$ for any $1 \leq j<n$ and any point $y$ in the ball of radius $2 \delta_{1} \sigma^{j / 2}$ around $f^{n-j}(x)$.

Proof. Since $n$ is a $(\sigma, \delta)$-hyperbolic time for $x$ we have $\operatorname{dist}_{\delta}\left(f^{n-j}(x), \mathcal{C}\right) \geq \sigma^{j}$ for any $1 \leq j<n$. According to the definition of the truncated distance, this means that

$$
\operatorname{dist}\left(f^{n-j}(x), \mathcal{C}\right)=\operatorname{dist}_{\delta}\left(f^{n-j}(x), \mathcal{C}\right) \geq \sigma^{b j} \quad \text { or else } \quad \operatorname{dist}\left(f^{n-j}(x), \mathcal{C}\right) \geq \delta .
$$

In either case, we have $\operatorname{dist}\left(y, f^{n-j}(x)\right)<\operatorname{dist}\left(f^{n-j}(x), \mathcal{C}\right) / 2$ for any $1 \leq j<n$, because we chose $b<1 / 2$ and $\delta_{1}<\delta / 4<1 / 4$. Therefore, we may use $\left(\mathrm{s}_{2}\right)$ to conclude that

$$
\log \frac{\left\|D f(y)^{-1}\right\|}{\left\|D f\left(f^{n-j}(x)\right)^{-1}\right\|} \leq B \frac{\operatorname{dist}\left(y, f^{n-j}(x)\right)}{\left.\operatorname{dist}\left(f^{n-j}(x)\right), \mathcal{C}\right)^{\beta}} \leq B \frac{2 \delta_{1} \sigma^{j / 2}}{\min \left\{\sigma^{b \beta j}, \delta^{\beta}\right\}} .
$$

Since $\delta$ and $\sigma$ are smaller than 1 , and we took $b \beta<1 / 2$, the term on the right hand side is bounded by $2 B \delta_{1} \delta^{-\beta}$. Moreover, our second condition on $\delta_{1}$ means that this last expression is smaller than $\log \sigma^{-1 / 2}$.

Proposition 3.3. Let $0<\sigma<1$ and $\delta>0$. If $n$ is a $(\sigma, \delta)$-hyperbolic time for $x$, then there exists a neighborhood $V_{n}$ of $x$ such that:

(1) $f^{n}$ maps $V_{n}$ diffeomorphically onto the ball of radius $\delta_{1}$ around $f^{n}(x)$;

(2) for each $x \in V_{n}$ we have $\left\|D f^{n}(x)^{-1}\right\| \leq \sigma^{n / 2}$;

(3) for all $1 \leq k<n$ and $y, z \in V_{n}$,

$$
\operatorname{dist}\left(f^{n-k}(y), f^{n-k}(z)\right) \leq \sigma^{k / 2} \operatorname{dist}\left(f^{n}(y), f^{n}(z)\right) .
$$

Proof. See [3, Lemma 5.2].

We shall refer to the sets $V_{n}$ as hyperbolic pre-balls and to their images $f^{n}\left(V_{n}\right)$ as hyperbolic balls. Notice that the latter are indeed balls of radius $\delta_{1}>0$.

Lemma 3.4. Given $0<c_{1}<c_{2}<A$ let $\theta=\left(c_{2}-c_{1}\right) /\left(A-c_{1}\right)$. Take $a_{1} \leq A, \ldots, a_{N} \leq A$ such that $\sum_{j=1}^{N} a_{j} \geq c_{2} N$. Then there are $l>\theta N$ and $1<n_{1}<\cdots<n_{l} \leq N$ so that $\sum_{j=n+1}^{n_{i}} a_{j} \geq c_{1}\left(n_{i}-n\right)$ for every $0 \leq n<n_{i}$ and $i=1, \ldots, l$. 
Proof. See [3, Lemma 3.1].

We say that the frequency of $(\sigma, \delta)$-hyperbolic times for $x \in M$ is bigger than $\theta>0$ if, for large $n \in \mathbb{N}$, there are $\ell \geq \theta n$ and integers $1 \leq n_{1}<n_{2} \cdots<n_{\ell} \leq n$ which are $(\sigma, \delta)$-hyperbolic times for $x$.

Proposition 3.5. Assume that $f: M \rightarrow M$ is non-uniformly expanding. Then there are $0<\sigma<1, \delta>0$ and $\theta>0$ (depending only on $\lambda$ and on the derivative of $f$ ) such that the frequency of $(\sigma, \delta)$-hyperbolic times for Lebesgue almost all $x \in M$ is bigger than $\theta$.

Proof. Assuming that (11) holds for $x \in M$, then for large $N \in \mathbb{N}$ we have

$$
\sum_{j=0}^{N-1}-\log \left\|D f\left(f^{j}(x)\right)^{-1}\right\| \geq \lambda N
$$

Take $\beta>0$ given by Definition [1.1, and fix any $\rho>\beta$. Then $\left(\mathrm{s}_{2}\right)$ implies that

$$
\left|\log \left\|D f(x)^{-1}\right\|\right| \leq \rho|\log \operatorname{dist}(x, \mathcal{C})|
$$

for every $x$ in a neighborhood $V$ of $\mathcal{C}$. Fix $\varepsilon_{1}>0$ so that $\rho \varepsilon_{1} \leq \lambda / 2$, and let $r_{1}>0$ be so that

$$
\sum_{j=0}^{N-1} \log \operatorname{dist}_{r_{1}}\left(f^{j}(x), \mathcal{C}\right) \geq-\varepsilon_{1} N
$$

The assumption of slow recurrence to the critical set ensures that this is possible. Fix any $K_{1} \geq \rho\left|\log r_{1}\right|$ large enough so that it is also an upper bound for $-\log \left\|D f^{-1}\right\|$ on the complement of $V$. Then let $J$ be the subset of times $1 \leq j \leq N$ such that $-\log \left\|D f\left(f^{j-1}(x)\right)^{-1}\right\|>K_{1}$, and define

$$
a_{j}= \begin{cases}-\log \left\|D f\left(f^{j-1}(x)\right)^{-1}\right\| & \text { if } j \notin J \\ 0 & \text { if } j \in J .\end{cases}
$$

By construction, $a_{j} \leq K_{1}$ for $1 \leq j \leq N$. Note that if $j \in J$ then $f^{j-1}(x) \in V$. Moreover, for each $j \in J$

$$
\rho\left|\log r_{1}\right| \leq K_{1}<-\log \left\|D f\left(f^{j-1}(x)\right)^{-1}\right\|<\rho\left|\log \operatorname{dist}\left(f^{j-1}(x), \mathcal{C}\right)\right|,
$$

which shows that $\operatorname{dist}\left(f^{j-1}(x), \mathcal{C}\right)<r_{1}$ for every $j \in J$. In particular,

$$
\operatorname{dist}_{r_{1}}\left(f^{j-1}(x), \mathcal{C}\right)=\operatorname{dist}\left(f^{j-1}(x), \mathcal{C}\right)<r_{1}, \quad \text { for all } j \in J .
$$

Therefore, by (101) and (111),

$$
\sum_{j \in J}-\log \left\|D f\left(f^{j-1}(x)\right)^{-1}\right\| \leq \rho \sum_{j \in J}\left|\log \operatorname{dist}\left(f^{j-1}(x), \mathcal{C}\right)\right| \leq \rho \varepsilon_{1} N .
$$

We have chosen $\varepsilon_{1}>0$ in such a way that the last term is less than $\lambda N / 2$. As a consequence,

$$
\sum_{j=1}^{N} a_{j}=\sum_{j=1}^{N}-\log \left\|D f\left(f^{j-1}(x)\right)^{-1}\right\|-\sum_{j \in J}-\log \left\|D f\left(f^{j-1}(x)\right)^{-1}\right\| \geq \frac{\lambda}{2} N .
$$

Thus, we have checked that we may apply Lemma 3.4 to the numbers $a_{1}, \ldots, a_{N}$, with $c_{1}=\lambda / 4, c_{2}=\lambda / 2$, and $A=K_{1}$. The lemma provides $\theta_{1}>0$ and $l_{1} \geq \theta_{1} N$ times $1 \leq p_{1}<\cdots<p_{l_{1}} \leq N$ such that

$$
\sum_{j=n+1}^{p_{i}}-\log \left\|D f\left(f^{j-1}(x)\right)^{-1}\right\| \geq \sum_{j=n+1}^{p_{i}} a_{j} \geq \frac{\lambda}{4}\left(p_{i}-n\right)
$$


for every $0 \leq n<p_{i}$ and $1 \leq i \leq l_{1}$.

Now fix $\varepsilon_{2}>0$ small enough so that $\varepsilon_{2}<\theta_{1} b \lambda / 4$, and let $r_{2}>0$ be such that

$$
\sum_{j=0}^{N-1} \log \operatorname{dist}_{r_{2}}\left(f^{j}(x), \mathcal{C}\right) \geq-\varepsilon_{2} N \text {. }
$$

Let $c_{1}=-b \lambda / 4, c_{2}=-\varepsilon_{2}, A=0$, and

$$
\theta_{2}=\frac{c_{2}-c_{1}}{A-c_{1}}=1-\frac{4 \varepsilon_{2}}{b \lambda}
$$

Applying Lemma 3.4 to $a_{j}=\log \operatorname{dist}_{r_{2}}\left(f^{j-1}(x), \mathcal{C}\right)$, with $1 \leq j \leq N$, we conclude that there are $l_{2} \geq \theta_{2} N$ times $1 \leq q_{1}<\cdots<q_{l_{2}} \leq N$ such that

$$
\sum_{j=n}^{q_{i}-1} \log \operatorname{dist}_{r_{2}}\left(f^{j}(x), \mathcal{C}\right) \geq-\frac{b \lambda}{4}\left(q_{i}-n\right)
$$

for every $0 \leq n<q_{i}$ and $1 \leq i \leq l_{2}$.

Finally, our condition on $\varepsilon_{2}$ means that $\theta_{1}+\theta_{2}>1$. Let $\theta=\theta_{1}+\theta_{2}-1$. Then there exist $l=\left(l_{1}+l_{2}-N\right) \geq \theta N$ times $1 \leq n_{1}<\cdots<n_{l} \leq N$ at which (12) and (14) occur simultaneously:

$$
\sum_{j=n}^{n_{i}-1}-\log \left\|D f\left(f^{j}(x)\right)^{-1}\right\| \geq \frac{\lambda}{4}\left(n_{i}-n\right)
$$

and

$$
\sum_{j=n}^{n_{i}-1} \log \operatorname{dist}_{r_{2}}\left(f^{j}(x), \mathcal{C}\right) \geq-\frac{b \lambda}{4}\left(n_{i}-n\right)
$$

for every $0 \leq n<n_{i}$ and $1 \leq i \leq l$. Letting $\sigma=e^{-\lambda / 4}$ we easily obtain from the inequalities above

$$
\prod_{j=n_{i}-k}^{n_{i}-1}\left\|D f\left(f^{j}(x)\right)^{-1}\right\| \leq \sigma^{k} \quad \text { and } \quad \operatorname{dist}_{r_{2}}\left(f^{n_{i}-k}(x), \mathcal{C}\right) \geq \sigma^{b k}
$$

for every $1 \leq i \leq l$ and $1 \leq k \leq n_{i}$. In other words, all those $n_{i}$ are $(\sigma, \delta)$-hyperbolic times for $x$, with $\delta=r_{2}$.

Remark 3.6. From the proof of the previous proposition one easily sees that condition (2) in the definition of non-uniformly expanding map is not needed in all its strength for the proof work. Actually, we have only used (21) in (11) and (13). Hence, it is enough that (21) holds for $\varepsilon=\min \left\{\varepsilon_{1}, \varepsilon_{2}\right\}$ and $\delta=\max \left\{r_{1}, r_{2}\right\}$.

Remark 3.7. Observe that the proof of Proposition 3.5 also gives that if for some $x \in M$ and $N \in \mathbb{N}$

$$
\sum_{j=0}^{N-1}-\log \left\|D f\left(f^{j}(x)\right)^{-1}\right\| \geq \lambda N \quad \text { and } \quad \sum_{j=0}^{N-1} \log \operatorname{dist}_{\delta}\left(f^{j}(x), \mathcal{C}\right) \geq-\varepsilon N
$$

(where $\varepsilon$ and $\delta$ chosen as in Remark 3.6), then there exist $1 \leq n_{1}<\cdots<n_{l} \leq N$ with $l \geq \theta N$ such that $n_{i}$ is a $(\sigma, \delta)$-hyperbolic time for $x$ for every $1 \leq i \leq l$.

Corollary 3.8. There exists $C_{0}=C_{0}(B, \beta, b, \sigma)>0$ such that for every hyperbolic preball $V_{n}$ and every $y, z \in V_{n}$

$$
\log \frac{\left|\operatorname{det} D f^{n}(y)\right|}{\left|\operatorname{det} D f^{n}(z)\right|} \leq C_{0} \operatorname{dist}\left(f^{n}(y), f^{n}(z)\right) .
$$


Proof. It suffices to take $C_{0} \geq \sum_{k=1}^{\infty} 2^{\beta} B \sigma^{(1 / 2-b \beta) k}$; recall that $b \beta<1 / 2$.

Corollary 3.9. There exists $C_{1}=C_{1}\left(C_{0}\right)>0$ such that for every hyperbolic pre-ball $V_{n}$ and every $y, z \in V_{n}$

$$
\frac{1}{C_{1}} \leq \frac{\left|\operatorname{det} D f^{n}(y)\right|}{\left|\operatorname{det} D f^{n}(z)\right|} \leq C_{1} .
$$

Proof. Take $C_{1}=\exp \left(C_{0} D\right)$, where $D$ is the diameter of $M$.

We finish this section deriving an useful consequence of the existence of positive frequency of hyperbolic times.

Lemma 3.10. Let $A \subset M$ be a set with positive Lebesgue measure whose points have frequency of $(\sigma, \delta)$-hyperbolic times bigger than $\theta>0$. Then there is $n_{0} \in \mathbb{N}$ such that for $n \geq n_{0}$

$$
\frac{1}{n} \sum_{j=1}^{n} \frac{m\left(A \cap H_{j}\right)}{m(A)} \geq \frac{\theta}{2},
$$

where $H_{j}$ is the set of points that have $j$ as a $(\sigma, \delta)$-hyperbolic time.

Proof. Since we are assuming that points in $A$ have frequency of $(\sigma, \delta)$-hyperbolic times bigger than $\theta>0$, then there are $n_{0} \in \mathbb{N}$ and a set $B \subset A$ with $m(B) \geq m(A) / 2$ such that for every $x \in B$ and $n \geq n_{0}$ there are $(\sigma, \delta)$-hyperbolic times $0<n_{1}<n_{2}<\cdots<n_{\ell} \leq n$ for $x$ with $\ell \geq \theta n$. Take now $n \geq n_{0}$ and let $\xi_{n}$ be the measure in $\{1, \ldots, n\}$ defined by $\xi_{n}(J)=\# J / n$, for each subset $J$. Then, using Fubini's Theorem

$$
\begin{aligned}
\frac{1}{n} \sum_{j=1}^{n} m\left(B \cap H_{j}\right) & =\int\left(\int_{B} \mathbf{1}(x, i) d m(x)\right) d \xi_{n}(i) \\
& =\int_{B}\left(\int \mathbf{1}(x, i) d \xi_{n}(i)\right) d m(x),
\end{aligned}
$$

where $\mathbf{1}(x, i)=1$ if $x \in H_{i}$, and $\mathbf{1}(x, i)=0$ otherwise. Since for every $x \in B$ and $n \geq n_{0}$ there are $0<n_{1}<n_{2}<\cdots<n_{\ell} \leq n$ with $\ell \geq \theta n$ such that $x \in H_{n_{i}}$ for $1 \leq i \leq \ell$, then the integral with respect to $d \xi_{n}$ is larger than $\theta$. So, the last expression in the formula above is bounded from below by $\theta m(B) \geq \theta m(A) / 2$.

\section{Markov struCtures}

The aim of this section is to show that non-uniformly expanding transformations induce piecewise expanding Markovian return maps. This has been proved in [4] and we follow the proof therein. Detailed proofs of most results are presented here in order to show how constants depend on one another.

Theorem 4.1. Let $f: M \rightarrow M$ be a $C^{2}$ non-uniformly expanding transitive transformation. Then $f$ induces some piecewise expanding Markovian return map on a ball $\Delta \subset M$. Moreover, if there exist $C, \gamma>0$ such that $m\left(\Gamma_{n}\right) \leq C n^{-\gamma}$, then there is $C^{\prime}>0$ such that the return time function satisfies $m\{R>n\} \leq C^{\prime} n^{-\gamma}$.

Assuming that $f$ is a non-uniformly expanding map, then by Proposition 3.5 there are $\sigma, \delta$ and $\theta$ such that Lebesgue almost every $x \in M$ has frequency of $(\sigma, \delta)$-hyperbolic times greater $\operatorname{than} \theta$. From the transitivity of $f$ and by [4, Lemma 2.5] we may fix $p \in M$ and $N_{0} \in \mathbb{N}$ for which

$$
\cup_{j=0}^{N_{0}} f^{-j}\{p\} \text { is } \delta_{1} / 3 \text {-dense in } M \text { and disjoint from } \mathcal{C},
$$


where $\delta_{1}>0$ is the radius of hyperbolic balls given by Proposition 3.3. Take constants $\varepsilon>0$ and $\delta_{0}>0$ so that

$$
\sqrt{\delta}_{0} \ll \delta_{1} / 2 \text { and } 0<\varepsilon \ll \delta_{0} .
$$

Let us introduce a couple of auxiliary lemmas.

Lemma 4.2. There are constants $K_{0}, D_{0}>0$ depending only on $f, \sigma, \delta_{1}$ and the point $p$, such that for any ball $B \subset M$ of radius $\delta_{1}$ there are an open set $V \subset B$ and an integer $0 \leq m \leq N_{0}$ for which:

(1) $f^{m}$ maps $V$ diffeomorphically onto $B\left(p, 2 \sqrt{\delta}_{0}\right)$;

(2) for each $x, y \in V$

$$
\log \left|\frac{\operatorname{det} D f^{m}(x)}{\operatorname{det} D f^{m}(y)}\right| \leq D_{0} \operatorname{dist}\left(f^{m}(x), f^{m}(y)\right) \text {. }
$$

Moreover, for each $0 \leq j \leq N_{0}$ the $j$-preimages of $B\left(p, 2 \sqrt{\delta}_{0}\right)$ are all disjoint from $\mathcal{C}$, and for $x$ belonging to any such $j$-preimage we have $K_{0}{ }^{-1} \leq\left\|D f^{j}(x)\right\| \leq K_{0}$.

Proof. Since $\cup_{j=0}^{N_{0}} f^{-j}\{p\}$ is $\delta_{1} / 3$ dense in $M$ and disjoint from $\mathcal{C}$, choosing $\delta_{0}>0$ sufficiently small we have that each connected component of the preimages of $B\left(p, 2 \sqrt{\delta}_{0}\right)$ up to time $N_{0}$ are bounded away from the critical set $\mathcal{C}$ and are contained in a ball of radius $\delta_{1} / 3$. This immediately implies that any ball $B \subset M$ of radius $\delta_{1}$ contains a preimage $V$ of $B\left(p, 2 \sqrt{\delta}_{0}\right)$ which is mapped diffeomorphically onto $B\left(p, 2 \sqrt{\delta}_{0}\right)$ in at most $N_{0}$ iterates. Moreover, since the number of iterations and the distance to the critical region are uniformly bounded, the volume distortion is uniformly bounded.

Observe that $\delta_{0}$ and $N_{0}$ have been chosen in such a way that all the connected components of the preimages of $B\left(p, 2 \sqrt{\delta}_{0}\right)$ up to time $N_{0}$ are uniformly bounded away from the critical set $\mathcal{C}$, and so there is some constant $K_{0}>1$ such that $K_{0}{ }^{-1} \leq\left\|D f^{m}(x)\right\| \leq K_{0}$ for all $1 \leq m \leq N_{0}$ and $x$ belonging to an $m$-preimage of $B\left(p, 2 \sqrt{\delta}_{0}\right)$.

Lemma 4.3. There exists $N_{\varepsilon}>0$ such that any ball $B \subset M$ of radius $\varepsilon$ contains a hyperbolic pre-ball $V_{n} \subset B$ with $n \leq N_{\varepsilon}$.

Proof. Take any $\varepsilon>0$ and a ball $B(z, \varepsilon)$. By Proposition 3.3 we may choose $n_{\varepsilon} \in \mathbb{N}$ large enough so that any hyperbolic pre-ball $V_{n}$ associated to a hyperbolic time $n \geq n_{\varepsilon}$ has diameter not exceeding $\varepsilon / 2$. Now notice that by Proposition 3.5 Lebesgue almost every point has an infinite number of hyperbolic times and therefore

$$
m\left(M \backslash \bigcup_{j=n_{\varepsilon}}^{n} H_{j}\right) \rightarrow 0 \quad \text { as } n \rightarrow \infty .
$$

Hence, it is possible to choose $N_{\varepsilon} \in \mathbb{N}$ such that

$$
m\left(M \backslash \bigcup_{j=n_{\varepsilon}}^{N_{\varepsilon}} H_{j}\right)<m(B(z, \varepsilon / 2)) .
$$

This ensures that there is a point $\hat{x} \in B(z, \varepsilon / 2)$ with a hyperbolic time $n \leq N_{\varepsilon}$ and associated hyperbolic pre-ball $V_{n}(x)$ contained in $B(z, \varepsilon)$.

Remark 4.4. Observe that if $n$ is a hyperbolic time for $f$, then $n$ is also a hyperbolic time for every map in a sufficiently small $C^{1}$ neighborhood of $f$. Hence, for given $\varepsilon>0$ the integer $N_{\varepsilon}$ may be taken uniform in a whole $C^{1}$ neighborhood of $f$, and only depending on $\varepsilon, \sigma$ and $\delta_{1}$. 
4.1. The partitioning algorithm. Here we describe the construction of the partition $(\bmod 0)$ of $\Delta_{0}=B\left(p, \delta_{0}\right)$. We introduce neighborhoods of $p$

$$
\Delta_{0}^{0}=B\left(p, \delta_{0}\right), \quad \Delta_{0}^{1}=B\left(p, 2 \delta_{0}\right), \quad \Delta_{0}^{2}=B\left(p, \sqrt{\delta}_{0}\right) \quad \text { and } \quad \Delta_{0}^{3}=B\left(p, 2 \sqrt{\delta}_{0}\right) .
$$

For $0<\sigma<1$ given by Proposition 3.5, let

$$
I_{k}=\left\{x \in \Delta_{0}^{1}: \delta_{0}\left(1+\sigma^{k / 2}\right)<\operatorname{dist}(x, p)<\delta_{0}\left(1+\sigma^{(k-1) / 2}\right)\right\}, \quad k \geq 1,
$$

be a partition $(\bmod 0)$ into countably many rings of $\Delta_{0}^{1} \backslash \Delta_{0}$. The construction of the partition of $\Delta_{0}$ is inductive and we describe precisely the general step of the induction below.

Take $R_{0}$ some large integer to be determined latter; we ignore any dynamics occurring up to time $R_{0}$. Assume that sets $\Delta_{i}, A_{i}, A_{i}^{\varepsilon} B_{i},\{R=i\}$ and functions $t_{i}: \Delta_{i} \rightarrow \mathbb{N}$ are defined for all $i \leq n-1$. For $i \leq R_{0}$ we just let $A_{i}=A_{i}^{\varepsilon}=\Delta_{i}=\Delta_{0}, B_{i}=\{R=i\}=\emptyset$ and $t_{i} \equiv 0$. Now let $\left(U_{n, j}^{3}\right)_{j}$ be the connected components of $f^{-n}\left(\Delta_{0}\right) \cap A_{n-1}^{\varepsilon}$ contained in hyperbolic pre-balls $V_{m}$, with $n-N_{0} \leq m \leq n$, which are mapped onto $\Delta_{0}^{3}$ by $f^{n}$. Take

$$
U_{n, j}^{i}=U_{n, j}^{3} \cap f^{-n} \Delta_{0}^{i}, \quad i=0,1,2,
$$

and set $R(x)=n$ for $x \in U_{n, j}^{0}$. Take also

$$
\Delta_{n}=\Delta_{n-1} \backslash\{R=n\} .
$$

The definition of the function $t_{n}: \Delta_{n} \rightarrow \mathbb{N}$ is slightly different in the general case:

$$
t_{n}(x)= \begin{cases}s & \text { if } x \in U_{n, j}^{1} \backslash U_{n, j}^{0} \text { and } f^{n}(x) \in I_{s} \text { for some } j \\ 0 & \text { if } x \in A_{n-1} \backslash \bigcup_{j} U_{n, j}^{1} \\ t_{n-1}(x)-1 & \text { if } x \in B_{n-1} \backslash \bigcup_{j} U_{n, j}^{1}\end{cases}
$$

Finally let

$$
A_{n}=\left\{x \in \Delta_{n}: t_{n}(x)=0\right\}, \quad B_{n}=\left\{x \in \Delta_{n}: t_{n}(x)>0\right\}
$$

and

$$
A_{n}^{\varepsilon}=\left\{x \in \Delta_{n}: \operatorname{dist}\left(f^{n+1}(x), f^{n+1}\left(A_{n}\right)\right)<\varepsilon\right\} .
$$

At this point we have completely described the inductive construction of the sets $A_{n}, A_{n}^{\varepsilon}$, $B_{n}$ and $\{R=n\}$.

The construction detailed before provides an algorithm for the definition of a family of topological balls contained in $\Delta_{0}$ and satisfying the Markov property as required. This algorithm does indeed produce a partition mod 0 of $\Delta_{0}$; see [4, Lemma 3.1].

Associated to each component $U_{n-k}^{0}$ of $\{R=n-k\}$, for some $k>0$, we have a collar $U_{n-k}^{1} \backslash U_{n-k}^{0}$ around it; knowing that the new components of $\{R=n\}$ do not intersect "too much" $U_{n-k}^{1} \backslash U_{n-k}^{0}$ is important for preventing overlaps on sets of the partition. This is indeed the case as long as $\varepsilon>0$ is taken small enough.

Lemma 4.5. If $\varepsilon>0$ is sufficiently small, then $U_{n}^{1} \cap\left\{t_{n-1} \geq 1\right\}=\emptyset$ for each $U_{n}^{1}$.

Proof. Take some $k>0$ and let $U_{n-k}^{0}$ be a component of $\{R=n-k\}$. Let $Q_{k}$ be the part of $U_{n-k}^{1}$ that is mapped by $f^{n-k}$ onto $I_{k}$ and assume that $Q_{k}$ intersects some $U_{n}^{3}$. Recall that, by construction, $Q_{k}$ is precisely the part of $U_{n-k}^{1}$ on which $t_{n-1}$ takes the value 1 . Letting $q_{1}$ and $q_{2}$ be any two points in distinct components (inner and outer) of the boundary of $Q_{k}$, we have by Proposition 3.3 and Lemma 4.2

$$
\operatorname{dist}\left(f^{n-k}\left(q_{1}\right), f^{n-k}\left(q_{2}\right)\right) \leq K_{0} \sigma^{\left(k-N_{0}\right) / 2} \operatorname{dist}\left(f^{n}\left(q_{1}\right), f^{n}\left(q_{2}\right)\right) .
$$


We also have

$$
\begin{aligned}
\operatorname{dist}\left(f^{n-k}\left(q_{1}\right), f^{n-k}\left(q_{2}\right)\right) & \geq \delta_{0}\left(1+\sigma^{(k-1) / 2}\right)-\delta_{0}\left(1+\sigma^{k / 2}\right) \\
& =\delta_{0} \sigma^{k / 2}\left(\sigma^{-1 / 2}-1\right),
\end{aligned}
$$

which combined with (16) gives

$$
\operatorname{dist}\left(f^{n}\left(q_{1}\right), f^{n}\left(q_{2}\right)\right) \geq K_{0}^{-1} \sigma^{N_{0} / 2} \delta_{0}\left(\sigma^{-1 / 2}-1\right) .
$$

On the other hand, since $U_{n}^{3} \subset A_{n-1}^{\varepsilon}$ by construction of $U_{n}^{3}$, taking

$$
\varepsilon<K_{0}^{-1} \sigma^{N_{0} / 2} \delta_{0}\left(\sigma^{-1 / 2}-1\right)
$$

we have $U_{n}^{3} \cap\left\{t_{n-1}>1\right\}=\emptyset$. This implies $U_{n}^{1} \cap\left\{t_{n-1} \geq 1\right\}=\emptyset$.

4.2. Expansion. Recall that by construction, the return time $R$ for an element $U$ of the partition $\mathcal{P}$ of $\Delta_{0}$ is formed by a certain number $n$ of iterations given by the hyperbolic time of a hyperbolic pre-ball $V_{n} \supset U$, and a certain number $m \leq N_{0}$ of additional iterates which is the time it takes to go from $f^{n}\left(V_{n}\right)$ which could be anywhere in $M$, to $f^{n+m}\left(V_{n}\right)$ which covers $\Delta_{0}$ completely. It follows from Proposition 3.3 and Lemma 4.2 that

$$
\left\|D f^{n+m}(x)^{-1}\right\| \leq\left\|D f^{m}\left(f^{n}(x)\right)^{-1}\right\| \cdot\left\|D f^{n}(x)^{-1}\right\|<K_{0} \sigma^{n / 2} \leq K_{0} \sigma^{\left(R_{0}-N_{0}\right) / 2} .
$$

By taking $R_{0}$ sufficiently large we can make this last expression smaller than 1.

4.3. Bounded distortion. For the bounded distortion estimate in Definition 2.1] we need to show that there exists a constant $K>0$ such that for any $x, y$ belonging to an element $U \in \mathcal{P}$ with return time $R$, we have

$$
\log \left|\frac{\operatorname{det} D f^{R}(x)}{\operatorname{det} D f^{R}(y)}\right| \leq K \operatorname{dist}\left(f^{R}(x), f^{R}(y)\right) .
$$

Recall that by construction, the return time $R$ for an element $U$ of the partition $\mathcal{P}$ of $\Delta_{0}$ is formed by a certain number $n$ of iterations given by the hyperbolic time of a hyperbolic pre-ball $V_{n} \supset U$, and a certain number $m=R-n \leq N_{0}$ of additional iterates which is the time it takes to go from $f^{n}\left(V_{n}\right)$ to $\Delta_{0}$ and cover it completely. By the chain rule

$$
\log \left|\frac{\operatorname{det} D f^{R}(x)}{\operatorname{det} D f^{R}(y)}\right|=\log \left|\frac{\operatorname{det} D f^{R-n}\left(f^{n}(x)\right)}{\operatorname{det} D f^{R-n}\left(f^{n}(y)\right)}\right|+\log \left|\frac{\operatorname{det} D f^{n}(x)}{\operatorname{det} D f^{n}(y)}\right| .
$$

For the first term in this last sum we observe that by Lemma 4.2 we have

$$
\log \left|\frac{\operatorname{det} D f^{R-n}\left(f^{n}(x)\right)}{\operatorname{det} D f^{R-n}\left(f^{n}(y)\right)}\right| \leq D_{0} \operatorname{dist}\left(f^{R}(x), f^{R}(y)\right) .
$$

For the second term in the sum above, we may apply Corollary 3.8 and obtain

$$
\log \left|\frac{\operatorname{det} D f^{n}(x)}{\operatorname{det} D f^{n}(y)}\right| \leq C_{0} \operatorname{dist}\left(f^{n}(x), f^{n}(y)\right) .
$$

Also by Lemma 4.2 we may write

$$
\operatorname{dist}\left(f^{n}(x), f^{n}(y)\right) \leq K_{0} \operatorname{dist}\left(f^{R}(x), f^{R}(y)\right) .
$$

Thus we just have to take $K=D_{0}+C_{0} K_{0}$.

In the next lemma we show that the bounded distortion condition in [5] is satisfied in our context. 
Lemma 4.6. For each $U \in \mathcal{P}$ we have

$$
\frac{\left\|D\left(J \circ\left(\left.F\right|_{U}\right)^{-1}\right)\right\|}{\left|J \circ\left(\left.F\right|_{U}\right)^{-1}\right|}<K
$$

where $J=\operatorname{det} D F$ is the Jacobian of $F$.

Proof. For simplicity we assume $\Delta \subset \mathbb{R}^{d}$. Observe that

$$
\frac{\left\|D\left(J \circ\left(\left.F\right|_{U}\right)^{-1}\right)\right\|}{\left|\left(J \circ\left(\left.F\right|_{U}\right)^{-1}\right)\right|}=\left\|D\left(\log \left|J \circ\left(\left.F\right|_{U}\right)^{-1}\right|\right)\right\| .
$$

Thus we just have to prove that the functions $\log \left|J \circ\left(\left.F\right|_{U}\right)^{-1}\right|, U \in \mathcal{P}$, have derivatives uniformly bounded by $K$. Take any point $x$ in the interior of $\Delta$ and $v$ a vector of the canonical basis of $\mathbb{R}^{d}$. By the bounded distortion condition of Definition 2.1 we have for small $t \in \mathbb{R}$

$$
\begin{aligned}
\log \left|J \circ\left(\left.F\right|_{U}\right)^{-1}\right|(x+t v) & -\log \left|J \circ\left(\left.F\right|_{U}\right)^{-1}\right|(x) \\
& \leq K \operatorname{dist}\left(F\left(\left(\left.F\right|_{U}\right)^{-1}(x+t v)\right), F\left(\left(\left.F\right|_{U}\right)^{-1}(x)\right)\right) \\
& =K t .
\end{aligned}
$$

This implies the uniform bound on derivatives that we need.

4.4. Metric estimates. Now we prove that the construction performed above does indeed produce a partition of $\Delta_{0}$ as in the Theorem 4.1. modulo a zero Lebesgue measure subset. We split our argument into two parts.

4.4.1. Estimates derived from the construction. In this first part we obtain some estimates relating the Lebesgue measure of the sets $A_{n}, B_{n}$ and $\{R>n\}$ with the help of specific information extracted from the inductive construction we performed in Subsection 4.1 .

Lemma 4.7. There exists a constant $a_{0}>0$ (not depending on $\delta_{0}$ ) such that

$$
m\left(B_{n-1} \cap A_{n}\right) \geq a_{0} m\left(B_{n-1}\right)
$$

for every $n \geq 1$.

Proof. It is enough to see that this holds for each connected component of $B_{n-1}$ at a time. Let $C$ be a component of $B_{n-1}$ and $Q$ be its outer ring corresponding to $t_{n-1}=1$. Observe that by Lemma 4.5 we have $Q=C \cap A_{n}$. Moreover, there must be some $k<n$ and a component $U_{k}^{0}$ of $\{R=k\}$ such that $f^{k}$ maps $C$ diffeomorphically onto $\bigcup_{i=k}^{\infty} I_{i}$ and $Q$ onto $I_{k}$, both with distortion bounded by $C_{1}$ and $e^{D_{0} L}$, where $L$ is the diameter of $M$; cf. Corollary 3.9 and Lemma 4.2. Thus, it is sufficient to compare the Lebesgue measures of $\bigcup_{i=k}^{\infty} I_{i}$ and $I_{k}$. We have

$$
\frac{m\left(I_{k}\right)}{m\left(\bigcup_{i=k}^{\infty} I_{i}\right)} \approx \frac{\left[\delta_{0}\left(1+\sigma^{(k-1) / 2}\right)\right]^{d}-\left[\delta_{0}\left(1+\sigma^{k / 2}\right)\right]^{d}}{\left[\delta_{0}\left(1+\sigma^{(k-1) / 2}\right)\right]^{d}-\delta_{0}^{d}} \approx 1-\sigma^{1 / 2} .
$$

Clearly this proportion does not depend on $\delta_{0}$.

Lemma 4.8. There exist $b_{0}, c_{0}>0$ with $b_{0}+c_{0}<1$ such that for every $n \geq 1$

(1) $m\left(A_{n-1} \cap B_{n}\right) \leq b_{0} m\left(A_{n-1}\right)$;

(2) $m\left(A_{n-1} \cap\{R=n\}\right) \leq c_{0} m\left(A_{n-1}\right)$.

Moreover $b_{0} \rightarrow 0$ and $c_{0} \rightarrow 0$ as $\delta_{0} \rightarrow 0$. 
Proof. It is enough to prove these estimates for each neighborhood of a component $U_{n}^{0}$ of $\{R=n\}$. Observe that by construction we have $U_{n}^{3} \subset A_{n-1}^{\varepsilon}$, which means that $U_{n}^{2} \subset A_{n-1}$, because $\varepsilon<\delta_{0}<\sqrt{\delta}_{0}$. Using the distortion bounds of $f^{n}$ on $U_{n}^{3}$ given by Corollary 3.9 and Lemma 4.2 we obtain

$$
\frac{m\left(U_{n}^{1} \backslash U_{n}^{0}\right)}{m\left(U_{n}^{2} \backslash U_{n}^{1}\right)} \approx \frac{m\left(\Delta_{0}^{1} \backslash \Delta_{0}^{0}\right)}{m\left(\Delta_{0}^{2} \backslash \Delta_{0}^{1}\right)} \approx \frac{\delta_{0}^{d}}{\delta_{0}^{d / 2}} \ll 1,
$$

which gives the first estimate. Moreover,

$$
\frac{m\left(U_{n}^{0}\right)}{m\left(U_{n}^{2} \backslash U_{n}^{1}\right)} \approx \frac{m\left(\Delta_{0}^{0}\right)}{m\left(\Delta_{0}^{2} \backslash \Delta_{0}^{1}\right)} \approx \frac{\delta_{0}^{d}}{\delta_{0}^{d / 2}} \ll 1,
$$

and this gives the second one.

The next result asserts that a fixed proportion of $A_{n-1} \cap H_{n}$ gives rise to new elements of the partition within a finite number of steps (not depending on $n$ ).

Proposition 4.9. There exist $c_{1}>0$ and a positive integer $N=N(\varepsilon)$ such that

$$
m\left(\bigcup_{i=0}^{N}\{R=n+i\}\right) \geq c_{1} m\left(A_{n-1} \cap H_{n}\right)
$$

for every $n \geq 1$.

Proof. Take $r=5 \delta_{0} K_{0}^{N_{0}}$, where $N_{0}$ and $K_{0}$ are given by Lemma 4.2. Let $\left\{z_{j}\right\}$ be a maximal set in $f^{n}\left(A_{n-1} \cap H_{n}\right)$ with the property that $B\left(z_{j}, r\right)$ are pairwise disjoint. By maximality we have $\bigcup_{j} B\left(z_{j}, 2 r\right) \supset f^{n}\left(A_{n-1} \cap H_{n}\right)$. Let $x_{j}$ be a point in $H_{n}$ such that $f^{n}\left(x_{j}\right)=z_{j}$ and consider the hyperbolic pre-ball $V_{n}\left(x_{j}\right)$ associated to $x_{j}$. Observe that $f^{n}$ sends $V_{n}\left(x_{j}\right)$ diffeomorphically onto a ball of radius $\delta_{1}$ around $z_{j}$ as in Proposition 3.5. In what follows, given $B \subset B\left(z_{j}, \delta_{1}\right)$, we will simply denote $\left(f^{n} \mid V_{n}\left(x_{j}\right)\right)^{-1}(B)$ by $f^{-n}(B)$.

Our aim now is to prove that $f^{-n}\left(B\left(z_{j}, r\right)\right)$ contains some component of $\left\{R=n+k_{j}\right\}$ with $0 \leq k_{j} \leq N_{\varepsilon}+N_{0}$. We start by showing that

$$
t_{n+k_{j}} \mid f^{-n}\left(B\left(z_{j}, \varepsilon\right)\right)>0 \text { for some } 0 \leq k_{j} \leq N_{\varepsilon}+N_{0} .
$$

Assume by contradiction that $t_{n+k_{j}} \mid f^{-n}\left(B\left(z_{j}, \varepsilon\right)\right)=0$ for all $0 \leq k_{j} \leq N_{\varepsilon}+N_{0}$. This implies that $f^{-n}\left(B\left(z_{j}, \varepsilon\right)\right) \subset A_{n+k_{j}}^{\varepsilon}$ for all $0 \leq k_{j} \leq N_{\varepsilon}+N_{0}$. Using Lemma 4.3 we may find a hyperbolic pre-ball $V_{m} \subset B\left(z_{j}, \varepsilon\right)$ with $m \leq N_{\varepsilon}$. Now, since $f^{m}\left(V_{m}\right)$ is a ball $B$ of radius $\delta_{1}$ it follows from Lemma 4.2 that there is some $V \subset B$ and $m^{\prime} \leq N_{0}$ with $f^{m^{\prime}}(V)=\Delta_{0}$. Thus, taking $k_{j}=m+m^{\prime}$ we have that $0 \leq k_{j} \leq N_{\varepsilon}+N_{0}$ and $f^{-n}\left(V_{m}\right)$ is an element of $\left\{R=n+k_{j}\right\}$ inside $f^{-n}\left(B\left(z_{j}, \varepsilon\right)\right)$. This contradicts the fact that $t_{n+k_{j}} \mid f^{-n}\left(B\left(z_{j}, \varepsilon\right)\right)=0$ for all $0 \leq k_{j} \leq N_{\varepsilon}+N_{0}$, and so (18) holds.

Let $k_{j}$ be the smallest integer $0 \leq k_{j} \leq N_{\varepsilon}+N_{0}$ for which $t_{n+k_{j}} \mid f^{-n}\left(B\left(z_{j}, \varepsilon\right)\right)>0$. Since $f^{-n}\left(B\left(z_{j}, \varepsilon\right)\right) \subset A_{n-1}^{\varepsilon} \subset\left\{t_{n-1} \leq 1\right\}$, there must be some element $U_{n+k_{j}}^{0}(j)$ of $\left\{R=n+k_{j}\right\}$ for which $f^{-n}\left(B\left(z_{j}, \varepsilon\right)\right) \cap U_{n+k_{j}}^{1}(j) \neq \emptyset$. Recall that by definition $f^{n+k_{j}}$ sends $U_{n+k_{j}}^{1}(j)$ diffeomorphically onto $\Delta_{0}^{1}$, the ball of radius $(1+s) \delta_{0}$ around $p$. From time $n$ to $n+k_{j}$ we may have some final "bad" period of length at most $N_{0}$ where the derivative of $f$ may contract, however being bounded from below by $1 / K_{0}$ in each step. Thus, the diameter of $f^{n}\left(U_{n+k_{j}}^{1}(j)\right)$ is at most $4 \delta_{0} K_{0}^{N_{0}}$. Since $B\left(z_{j}, \varepsilon\right)$ intersects $f^{n}\left(U_{n+k_{j}}^{1}(j)\right)$ and $\varepsilon<\delta_{0}<\delta_{0} K_{0}^{N_{0}}$, we have by the definition of $r$ that $f^{-n}\left(B\left(z_{j}, r\right)\right) \supset U_{n+k_{j}}^{0}(j)$. Thus we have shown that $f^{-n}\left(B\left(z_{j}, r\right)\right)$ contains some component of $\left\{R=n+k_{j}\right\}$ with 
$0 \leq k_{j} \leq N_{\varepsilon}+N_{0}$. Moreover, since $n$ is a hyperbolic time for $x_{j}$, we have by the distortion control given by Corollary 3.9

$$
\frac{m\left(f^{-n}\left(B\left(z_{j}, 2 r\right)\right)\right)}{m\left(f^{-n}\left(B\left(z_{j}, r\right)\right)\right)} \leq C_{1} \frac{m\left(B\left(z_{j}, 2 r\right)\right)}{m\left(B\left(z_{j}, r\right)\right)}
$$

and

$$
\frac{m\left(f^{-n}\left(B\left(z_{j}, r\right)\right)\right)}{m\left(U_{n+k_{j}}^{0}(j)\right)} \leq C_{0} \frac{m\left(B\left(z_{j}, r\right)\right)}{m\left(f^{n}\left(U_{n+k_{j}}^{0}(j)\right)\right)} .
$$

Here we are implicitly assuming that

$$
r=r\left(\delta_{0}\right)<\delta_{1} / 2
$$

This can be done by taking $\delta_{0}$ small enough. Note that estimates on $N_{0}$ and $K_{0}$ improve when we diminish $\delta_{0}$.

From time $n$ to time $n+k_{j}$ we have at most $k_{j}=m_{1}+m_{2}$ iterates with $m_{1} \leq N_{\varepsilon}$, $m_{2} \leq N_{0}$ and $\left.f^{n}\left(U_{n+k_{j}}^{0}(j)\right)\right)$ containing some point $w_{j} \in H_{m_{1}}$. By the definition of $(\sigma, \delta)$ hyperbolic time we have $\operatorname{dist}_{\delta}\left(f^{i}(x), \mathcal{C}\right) \geq \sigma^{b N_{\varepsilon}}$ for every $0 \leq i \leq m_{1}$, which implies that there is some constant $D=D(\varepsilon)>0$ such that $\left|\operatorname{det}\left(D f^{i}(x)\right)\right| \leq D$ for $0 \leq i \leq m_{1}$ and $x \in f^{n}\left(U_{n+k_{j}}^{0}(j)\right)$. On the other hand, since the first $N_{0}$ preimages of $\Delta_{0}$ are uniformly bounded away from $\mathcal{C}$ we also have some $D^{\prime}>0$ such that $\left|\operatorname{det}\left(D f^{i}(x)\right)\right| \leq D^{\prime}$ for every $0 \leq i \leq m_{2}$ and $x$ belonging to an $i$ preimage of $\Delta_{0}$. Hence,

$$
m\left(f^{n}\left(U_{n+k_{j}}^{0}(j)\right)\right) \geq \frac{1}{D D^{\prime}} m\left(\Delta_{0}\right),
$$

which combined with (20) gives

$$
m\left(f^{-n}\left(B\left(z_{j}, r\right)\right)\right) \leq C m\left(U_{n+k_{j}}^{0}(j)\right),
$$

with $C$ only depending on $C_{1}, D, D^{\prime}, \delta_{0}$ and the dimension of $M$. We also deduce from (19) that

$$
m\left(f^{-n}\left(B\left(z_{j}, 2 r\right)\right)\right) \leq C^{\prime} m\left(f^{-n}\left(B\left(z_{j}, r\right)\right)\right)
$$

with $C^{\prime}$ only depending on $C_{1}$ and the dimension of $M$. Finally let us compare the Lebesgue measure of the sets $\bigcup_{i=0}^{N}\{R=n+i\}$ and $A_{n-1} \cap H_{n}$. We have

$$
m\left(A_{n-1} \cap H_{n}\right) \leq \sum_{j} m\left(f^{-n}\left(B\left(z_{j}, 2 r\right)\right)\right) \leq C^{\prime} \sum_{j} m\left(f^{-n}\left(B\left(z_{j}, r\right)\right)\right) .
$$

On the other hand, by the disjointness of the balls $B\left(z_{j}, r\right)$ we have

$$
\sum_{j} m\left(f^{-n}\left(B\left(z_{j}, r\right)\right)\right) \leq C \sum_{j} m\left(U_{n+k_{j}}^{0}(j)\right) \leq C m\left(\bigcup_{i=0}^{N}\{R=n+i\}\right) .
$$

We just have to take $c_{1}=\left(C C^{\prime}\right)^{-1}$.

Remark 4.10. It follows from the choice of the constants $D$ and $D^{\prime}$ (and so also $C$ and $C^{\prime}$ ) that the constant $c_{1}$ only depends on the constants $\sigma, b, N_{\varepsilon}, C_{1}$ and $N_{0}$. 
4.4.2. General estimates. For the time being we have taken a disk $\Delta_{0}$ of radius $\delta_{0}>0$ around a point $p$ and defined inductively the subsets $A_{n}, B_{n},\{R=n\}$ and $\Delta_{n}$ which are related in the following way:

$$
\Delta_{n}=\Delta_{0} \backslash\{R \leq n\}=A_{n} \dot{\cup} B_{n} .
$$

Since we are dealing with a non-uniformly expanding map, we also have defined for each $n \in \mathbb{N}$ the set $H_{n}$ of points that have $n$ as a $(\sigma, \delta)$-hyperbolic time, and the tail of expansion $\Gamma_{n}$ as in (6). From the definition of $\Gamma_{n}$, Remark 3.7 and Lemma 3.10 we deduce:

$\left(\mathrm{m}_{1}\right)$ there is $\theta>0$ such that for every $n \geq 1$ and every $A \subset M \backslash \Gamma_{n}$ with $m(A)>0$

$$
\frac{1}{n} \sum_{j=1}^{n} \frac{m\left(A \cap H_{j}\right)}{m(A)} \geq \theta .
$$

Moreover, we have proved in Lemma 4.7. Lemma 4.8 and Proposition 4.9 that the following metric relations also hold:

$\left(\mathrm{m}_{2}\right)$ there is $a_{0}>0$ (bounded away from 0 with $\left.\delta_{0}\right)$ such that for $n \geq 1$

$$
m\left(B_{n-1} \cap A_{n}\right) \geq a_{0} m\left(B_{n-1}\right) ;
$$

$\left(\mathrm{m}_{3}\right)$ there are $b_{0}, c_{0}>0$ with $b_{0}+c_{0}<1$ and $b_{0}, c_{0} \rightarrow 0$ as $\delta_{0} \rightarrow 0$, such that for $n \geq 1$

$$
\frac{m\left(A_{n-1} \cap B_{n}\right)}{m\left(A_{n-1}\right)} \leq b_{0} \quad \text { and } \quad \frac{m\left(A_{n-1} \cap\{R=n\}\right)}{m\left(A_{n-1}\right)} \leq c_{0} ;
$$

$\left(\mathrm{m}_{4}\right)$ there is $c_{1}>0$ and an integer $N \geq 0$ such that for $n \geq 1$

$$
m\left(\bigcup_{i=0}^{N}\{R=n+i\}\right) \geq c_{1} m\left(A_{n-1} \cap H_{n}\right) .
$$

In the inductive process of construction of the sets $A_{n}, B_{n},\{R=n\}$ and $\Delta_{n}$ we have fixed some large integer $R_{0}$, being this the first step at which the construction began. Recall that $A_{n}=\Delta_{n}=\Delta_{0}$ and $B_{n}=\{R=n\}=\emptyset$ for $n \leq R_{0}$. For technical reasons we will assume that

$$
R_{0}>\max \left\{2(N+1), \frac{12}{\theta}\right\} .
$$

Note that since $N$ and $\theta$ do not depend on $R_{0}$ this is always possible.

This is the abstract setting under which we will be completing the proof of Theorem 4.1 . From now on we will only make use of the metric relations $\left(m_{1}\right)-\left(m_{4}\right)$ and will not be concerned with any other properties about these sets.

Lemma 4.11. There is $a_{1}>0$, with $a_{1} \rightarrow 0$ as $\delta_{0} \rightarrow 0$, such that for all $n \geq 1$

$$
m\left(B_{n}\right) \leq a_{1} m\left(A_{n}\right)
$$

Proof. Let us just mention how the constant $a_{1}>0$ appears. By $\left(\mathrm{m}_{3}\right)$

$$
m\left(A_{n} \cap A_{n-1}\right) \geq \eta m\left(A_{n-1}\right),
$$

where $\eta=1-b_{0}-c_{0}$. Then we take

$$
\widehat{a}=\frac{b_{0}+c_{0}}{a_{0}} \text { and } \quad a_{1}=\frac{\left(1+a_{0}\right) b_{0}+c_{0}}{a_{0} \eta} .
$$

The proof now follows exactly as in 4, Proposition 5.4].

Corollary 4.12. There exists $c_{2}>0$ such that for every $n \geq 1$

$$
m\left(\Delta_{n}\right) \leq c_{2} m\left(\Delta_{n+1}\right)
$$


Proof. Using $\left(\mathrm{m}_{3}\right)$ we obtain

$$
m\left(\Delta_{n+1}\right) \geq m\left(A_{n+1}\right) \geq\left(1-b_{0}-c_{0}\right) m\left(A_{n}\right) .
$$

On the other hand, by Lemma 4.11.

$$
m\left(\Delta_{n}\right)=m\left(A_{n}\right)+m\left(B_{n}\right) \leq\left(1+a_{1}^{-1}\right) m\left(A_{n}\right) .
$$

It is enough to take $c_{2}=\left(1+a_{1}^{-1}\right) /\left(1-b_{0}-c_{0}\right)$.

At this point we are able to definitely specify the choice of $\delta_{0}$. First of all, let us recall that the number $\theta$ in $\left(\mathrm{m}_{1}\right)$ does not depend on $\delta_{0}$. Assume that $m\left(\Gamma_{n}\right) \leq C n^{-\gamma}$, for some $C, \gamma>0$, and pick $\alpha>0$ such that

$$
\alpha<\left(\frac{\theta}{12}\right)^{\gamma+1}
$$

Then we choose $\delta_{0}>0$ small enough so that

$$
a_{1}<2 \alpha .
$$

This is possible because $a_{1} \rightarrow 0$ as $\delta_{0} \rightarrow 0$ by Lemma 4.11 .

Since $m\left(\Delta_{n}\right)=m\left(A_{n}\right)+m\left(B_{n}\right)$, we easily deduce from $\left(\mathrm{m}_{4}\right)$ and Lemma 4.11 that if we take

$$
b_{1}=\frac{c_{1}}{1+a_{1}}
$$

then

$$
m\left(\cup_{i=0}^{N}\{R=n+i\}\right) \geq b_{1} \frac{m\left(A_{n-1} \cap H_{n}\right)}{m\left(A_{n-1}\right)} m\left(\Delta_{n-1}\right) .
$$

This immediately implies that

$$
m\left(\Delta_{n+N}\right) \leq\left(1-b_{1} \frac{m\left(A_{n-1} \cap H_{n}\right)}{m\left(A_{n-1}\right)}\right) m\left(\Delta_{n-1}\right) .
$$

At this point we obtained some recurrence relation for the Lebesgue measure of the sets $\Delta_{n}$. Since $\left(\Delta_{n}\right)_{n}$ forms a decreasing sequence of sets we finally have

$$
m\left(\Delta_{n+N}\right) \leq \exp \left(-\frac{b_{1}}{N+1} \sum_{j=R_{0}}^{n} \frac{m\left(A_{j-1} \cap H_{j}\right)}{m\left(A_{j-1}\right)}\right) m\left(\Delta_{0}\right) .
$$

We will complete the proof of Theorem 4.1 by considering several different cases, according to the behavior of the proportions $m\left(A_{j-1} \cap H_{j}\right) / m\left(A_{j-1}\right)$. We define for each $n \geq 1$

$$
E_{n}=\left\{j \leq n: \frac{m\left(A_{j-1} \cap H_{j}\right)}{m\left(A_{j-1}\right)}<\alpha\right\},
$$

and

$$
F=\left\{n \in \mathbb{N}: \frac{\# E_{n}}{n}>1-\frac{\theta}{12}\right\} .
$$

Proposition 4.13. Take any $n \in F$ with $n \geq R_{0}$. If $m\left(A_{n}\right) \geq 2 m\left(\Gamma_{n}\right)$, then there is some $0<k=k(n)<n$ for which $m\left(A_{n}\right)<(k / n)^{\gamma} m\left(A_{k}\right)$.

Proof. See [4, Proposition 6.1].

Let us now complete the proof of Theorem 4.1. From Lemma 4.11 we get

$$
m\left(\Delta_{n}\right) \leq\left(1+a_{1}\right) m\left(A_{n}\right) .
$$

Hence, it is enough to derive the tail estimate of Theorem 4.1 for $m\left(A_{n}\right)$ in the place of $m\{R>n\}=m\left(\Delta_{n}\right)$. Given any large integer $n$, we consider the following two cases: 
(1) If $n \in \mathbb{N} \backslash F$, then by (29) and Corollary 4.12 we have

$$
m\left(\Delta_{n}\right) \leq c_{2}^{N} \exp \left(-\frac{b_{1} \theta \alpha}{12(N+1)}\left(n-R_{0}\right)\right) m\left(\Delta_{0}\right) .
$$

(2) If $n \in F$, then we distinguish the next two subcases:

(a) If $m\left(A_{n}\right)<2 m\left(\Gamma_{n}\right)$, then nothing has to be done.

(b) If $m\left(A_{n}\right) \geq 2 m\left(\Gamma_{n}\right)$, then we apply Proposition 4.13 and get some $k_{1}<n$ for which

$$
m\left(A_{n}\right)<\left(\frac{k_{1}}{n}\right)^{\gamma} m\left(A_{k_{1}}\right) .
$$

The only case we are left to consider is $2(\mathrm{~b})$. In such case, either $k_{1}$ is in situation 1 or 2 (a), or by Proposition 4.13 we can find $k_{2}<k_{1}$ for which

$$
m\left(A_{k_{1}}\right)<\left(\frac{k_{2}}{k_{1}}\right)^{\gamma} m\left(A_{k_{2}}\right) .
$$

Arguing inductively we are able to show that there is a sequence of integers $0<k_{s}<$ $\cdots<k_{1}<n$ for which one of the following situations eventually holds:

$$
\text { (C) } m\left(A_{n}\right)<\left(\frac{R_{0}}{n}\right)^{\gamma} m\left(\Delta_{0}\right) \text {. }
$$

In all these three situations we arrive at the desired conclusion of Theorem 4.1. Situation (C) corresponds to falling in case 2(b) above successively until $k_{s} \leq R_{0}$.

\section{UNIFORMNESS}

Let us remark that the ball on which the piecewise expanding Markovian return map is defined may be taken the same for every map belonging to a sufficiently small $C^{2}$ neighborhood of a map $f$ in a uniform family. In fact, we have taken the ball $\Delta_{0}$ centered at a point $p \in M$ which has been chosen in (15). Since $\delta_{1}$ may be chosen the same for every $f$ in a uniform family, and the radius $\delta_{0}$ of the ball $\Delta_{0}$ may be taken uniform in a neighborhood of $f$ (see Remark [5.2), then the point $p$ and $N_{0}$, and hence the ball $\Delta_{0}$, may be taken the same for every map belonging to a sufficiently small $C^{2}$ neighborhood of $f$. Observe also that by an implicit function argument the critical set varies continuously with the map in the $C^{2}$ topology.

The construction of the Markovian return map in Section 4 can be performed in such a way that the following uniformity condition holds:

$\left(\mathrm{u}_{0}\right)$ given an integer $N \geq 1$ and $\epsilon>0$, there is $\delta=\delta(\epsilon, N)>0$ such that for $j=1, \ldots, N$

$$
\left\|f-f_{0}\right\|_{C^{k}}<\delta \Rightarrow m\left(\left\{R_{f}=j\right\} \triangle\left\{R_{f_{0}}=j\right\}\right)<\epsilon,
$$

where $\Delta$ represents the symmetric difference of two sets.

This is just by continuity of the inductive construction for maps in a $C^{k}$ neighborhood of the original map. In fact, the construction of the partition on which the map $R_{f}$ takes constant values is based on a finite number of iterations of the map $f$. By continuity, we can perform the construction of the partition in such a way that for some fixed integer $N$ the Lebesgue measure of $\left\{R_{f}=j\right\}$ varies continuously with the map $f$ for $j \leq N$. Moreover, the Lebesgue measures of the auxiliary sets $A_{j}$ and $B_{j}$ also vary continuously 
with the map $f$ for $j \leq N$. Hence, the construction can be carried out with $R_{f}$ depending continuously on $f$ as stated in $\left(\mathrm{u}_{0}\right)$.

Lemma 5.1. Assume $\left(u_{0}\right)$ holds for $f_{0}$. Suppose moreover that given any $\epsilon>0$ there are $N \geq 1$ and $\delta>0$ for which

$$
\left\|f-f_{0}\right\|_{C^{k}}<\delta \Rightarrow\left\|\sum_{j=N}^{\infty} \mathbf{1}_{\left\{R_{f}>j\right\}}\right\|_{1}<\epsilon
$$

Then uniformity condition $\left(u_{1}\right)$ holds for $f_{0}$.

Proof. For the sake of notational simplicity we shall write $R$ instead of $R_{f}$ and $R_{0}$ instead of $R_{f_{0}}$. We need to show that given $\epsilon>0$ there is $\delta>0$ such that for any $f \in \mathcal{F}$

$$
\left\|f-f_{0}\right\|_{C^{k}}<\delta \quad \Rightarrow \quad\left\|R-R_{0}\right\|_{1}<\epsilon
$$

Since

$$
R_{0}=\sum_{j=0}^{\infty} \mathbf{1}_{\left\{R_{0}>j\right\}} \quad \text { and } \quad R=\sum_{j=0}^{\infty} \mathbf{1}_{\{R>j\}}
$$

then we have

$$
\left\|R-R_{0}\right\|_{1} \leq \sum_{j=0}^{N-1}\left\|\mathbf{1}_{\left\{R_{0}>j\right\}}-\mathbf{1}_{\{R>j\}}\right\|_{1}+\left\|\sum_{j=N}^{\infty} \mathbf{1}_{\left\{R_{0}>j\right\}}\right\|_{1}+\left\|\sum_{j=N}^{\infty} \mathbf{1}_{\{R>j\}}\right\|_{1} .
$$

By $\left(\mathrm{u}_{0}\right)$ and (32) all these terms can be made small for $f$ close to $f_{0}$.

Let $\mathcal{F}$ be a uniform family of non-uniformly expanding maps. Given $f \in \mathcal{F}$ we let the expansion time function $\mathcal{E}^{f}$ and the recurrence time function $\mathcal{R}^{f}$ be defined as in (44) and (5) respectively. The tail of expansion $\Gamma_{n}^{f}$ is also defined for $f \in \mathcal{F}$ as in (6) for $n \geq 1$.

Lemma 5.2. Let $\mathcal{F}$ be a uniform family of $C^{k}(k \geq 2)$ non-uniformly maps for which there are $C>0$ and $\gamma>0$ such that $m\left(\Gamma_{n}^{f}\right) \leq C n^{-\gamma}$, for all $n \geq 1$ and $f \in \mathcal{F}$. Then the constant $C^{\prime}$ in Theorem 4.1 may be taken uniformly in a neighborhood of each $f \in \mathcal{F}$.

Proof. As one can easily see from case (B) in the last part of the previous section, the constant $C^{\prime}>0$ in Theorem 4.1] depends on the constant $C>0$. Moreover, from (30) and the three possible cases one sees that $C^{\prime}$ also depends on some previous constants, namely $\alpha, a_{1}, b_{1}, c_{1}, \theta, N$ and $R_{0}$. It is possible to check that all these constants ultimately depend on the constants $B, \beta, b$ and $\lambda$ associated to the non-uniformly expanding map $f$. Naturally they also depend on the first and second derivatives of $f$. We explicit the dependence of the various constants in the table below: 


\begin{tabular}{|c|c|c|}
\hline Constant & Dependence & Reference \\
\hline$\sigma, \delta, \theta$ & $\bar{\lambda}$ & Proposition 3.5 \\
\hline$\delta_{1}$ & $B, \beta, \sigma, \delta$ & Lemma 3.2 \\
\hline$\alpha$ & $\theta$ & (25) \\
\hline$N_{0}$ & $\delta_{1}$ & (15) \\
\hline$D_{0}, K_{0}$ & $\sigma, \delta_{1}$ & Lemma 4.2 \\
\hline$C_{0}$ & $B, \beta, b, \sigma$ & Corollary 3.8 \\
\hline$C_{1}$ & $C_{0}$ & Corollary 3.9 \\
\hline$\delta_{0}$ & $\delta_{1}, \alpha$ & Lemma 4.2, (21), (25) \\
\hline$a_{0}$ & $\sigma, C_{1}, D_{0}$ & Lemma 4.7 \\
\hline$b_{0}, c_{0}$ & $C_{1}, D_{0}, \delta_{0}$ & Lemma 4.8 \\
\hline$a_{1}$ & $a_{0}, b_{0}, c_{0}, \alpha$ & (24), (26) \\
\hline$c_{1}$ & $\sigma, b, N_{\varepsilon}, C_{0}, N_{0}$ & Remark 4.10 \\
\hline$b_{1}$ & $a_{1}, c_{1}$ & (27) \\
\hline$c_{2}$ & $a_{1}, b_{0}, c_{0}$ & Corollary 4.12 \\
\hline$\varepsilon$ & $K_{0}, N_{0}, \delta_{0}, \sigma$ & (17) \\
\hline$N_{\varepsilon}$ & $\varepsilon, \sigma, \delta_{1}$ & Remark 4.4 \\
\hline$N$ & $N_{0}, N_{\varepsilon}$ & Proposition 4.9 \\
\hline$R_{0}$ & $K_{0}, \sigma, N_{0}, N, \theta$ & Subsection 4.2 (22) \\
\hline
\end{tabular}

For better understanding dependencies we use the convention that no constant depends on a constant from a line below. Consequently we have all constants depending on $B, \beta$, $b$ and $\lambda$.

Proposition 5.3. Let $\mathcal{F}$ be a uniform family of $C^{k}(k \geq 2)$ non-uniformly maps for which there are $C>0$ and $\gamma>1$ such that $m\left(\Gamma_{n}^{f}\right) \leq C n^{-\gamma}$, for all $n \geq 1$ and $f \in \mathcal{F}$. Then conditions $\left(u_{1}\right)$ and $\left(u_{2}\right)$ hold for each $f \in \mathcal{F}$.

Proof. Take any $f_{0} \in \mathcal{F}$. If we assume that there are $C>0$ and $\gamma>1$ such that $m\left(\Gamma_{n}^{f}\right) \leq C n^{-\gamma}$ for all $n \geq 1$ and all $f \in \mathcal{F}$, then by Theorem 4.1 there is a constant $C^{\prime}>0$ such that $m\left\{R_{f}>j\right\} \leq C^{\prime} n^{-\gamma}$ for all $n \geq 1$ and all $f \in \mathcal{F}$, as long as $f$ is taken in a sufficiently small $C^{k}$ neighborhood of $f_{0}$ in $\mathcal{F}$, say $f \in \mathcal{F}$ with $\left\|f-f_{0}\right\|_{C^{k}}<\delta$. Actually, as we have observed in Remark 5.2 the constant $C^{\prime}$ may be taken uniformly in a neighborhood of the map $f_{0}$. Thus, given $f \in \mathcal{F}$ with $\left\|f-f_{0}\right\|_{C^{k}}<\delta$ and an integer $N \geq 1$, we have

$$
\left\|\sum_{j=N}^{\infty} \mathbf{1}_{\left\{R_{f}>j\right\}}\right\|_{1} \leq \sum_{j=N}^{\infty} m\left(\left\{R_{f}>j\right\}\right) \leq \sum_{j=N}^{\infty} C^{\prime} n^{-\gamma}
$$

Since we are assuming $\gamma>1$, this last sum can be made arbitrarily small if we take $N$ large enough. Applying Lemma 5.1 we obtain uniformity condition $\left(\mathrm{u}_{1}\right)$.

For proving that $\left(\mathrm{u}_{2}\right)$ holds, we have to show that the constants $\kappa$ and $K$ in Definition 2.1 may be chosen uniformly for $f$ in a $C^{k}$ neighborhood of $f_{0}$ in the uniform family $\mathcal{F}$. The constant $K$ is given in Subsection 44.3. As it has been shown there, it only depends on $C_{0}, D_{0}$ and $K_{0}$. From Remark 5.2 we see that these constants may be chosen uniformly in $\mathcal{F}$. On the other hand, the constant $\kappa$ appeared in Subsection 4.2 and depends on $\sigma$, $N_{0}, K_{0}$ and $R_{0}$, which again may be chosen uniformly in $\mathcal{F}$.

As a consequence of Proposition 5.3 and Theorem 2.2 we obtain Theorem $\mathrm{A}$ 


\section{An EXAMPLE}

Here we present robust ( $C^{1}$ open) classes of local diffeomorphisms (with no critical set) that are non-uniformly expanding. Such classes of maps were presented in [3], and can be obtained, e.g. through deformation of a uniformly expanding map by isotopy inside some small region. In general, these maps are not expanding: deformation can be made in such way that the new map has periodic saddles.

Let $M$ be any compact manifold supporting some uniformly expanding map $f_{0}$ : there exists $\sigma_{0}>1$ such that

$$
\left\|D f_{0}(x) v\right\|>\sigma_{0}\|v\| \quad \text { for every } x \in M \text { and } v \in T_{x} M .
$$

For instance, $M$ could be the $d$-dimensional torus $T^{d}$. Let $V \subset M$ be some small compact domain, so that $f_{0} \mid V$ is injective. Let $f$ be any map in a small $C^{1}$-neighborhood $\mathcal{N}$ of $f_{0}$ so that $\left\|D f(x)^{-1}\right\|<\sigma_{0}$ for every $x$ outside $V$. Assume moreover that the $C^{1}$-neighborhood sufficiently small in such a way that:

(1) $f$ is volume expanding everywhere: there is $\sigma_{1}>1$ such that

$$
|\operatorname{det} D f(x)|>\sigma_{1} \quad \text { for every } x \in M \text {; }
$$

(2) $f$ is not too contracting on $V$ : there is some small $\delta>0$ such that

$$
\left\|D f(x)^{-1}\right\|<1+\delta \quad \text { for every } x \in V .
$$

We are going to show that every map $f$ in such a $C^{1}$-neighborhood $\mathcal{N}$ of $f_{0}$ is nonuniformly expanding.

Lemma 6.1. Let $B_{1}, \ldots, B_{p}, B_{p+1}=V$ be any partition of $M$ into domains such that $f$ is injective on $B_{j}$, for $1 \leq j \leq p+1$. There exists $\theta>0$ (only depending on $f_{0}$ ) such that the orbit of Lebesgue almost every point $x \in M$ spends a fraction $\theta$ of the time in

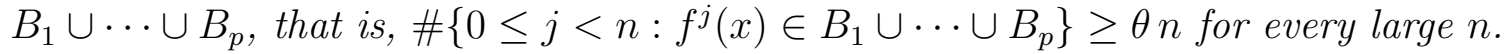

Proof. Let $n$ be fixed. Given a sequence $\underline{i}=\left(i_{0}, i_{1}, \ldots, i_{n-1}\right)$ in $\{1, \ldots, p+1\}$, we denote

$$
[\underline{i}]=B_{i_{0}} \cap f^{-1}\left(B_{i_{1}}\right) \cap \cdots \cap f^{-n+1}\left(B_{i_{n-1}}\right) .
$$

Moreover, we define $g(\underline{i})$ to be the number of values of $0 \leq j \leq n-1$ for which $i_{j} \leq p$. We begin by noting that, given any $\theta>0$, the total number of sequences $\underline{i}$ for which $g(\underline{i})<\theta n$ is bounded by

$$
\sum_{k<\theta n}\left(\begin{array}{c}
n \\
k
\end{array}\right) p^{k} \leq \sum_{k \leq \theta n}\left(\begin{array}{c}
n \\
k
\end{array}\right) p^{\theta n}
$$

A standard application of Stirling's formula (gives that the last expression is bounded by $e^{\gamma n} p^{\theta n}$, where $\gamma$ depends only on $\theta$ and goes to zero when $\theta$ goes to zero. On the other hand, since we are assuming that $f$ is volume expanding everywhere and not too contracting on $B_{p+1}$, we have $m([\underline{i}]) \leq m(M) \sigma_{1}^{-(1-\theta) n}$. Then the measure of the union $I_{n}$ of all the sets $[\underline{i}]$ with $g(\underline{i})<\theta n$ is less than $m(M) \sigma_{1}^{-(1-\theta) n} e^{\gamma n} p^{\theta n}$ Since $\sigma_{1}>1$, we may fix $\theta$ small so that $e^{\gamma} p^{\theta}<\sigma_{1}^{1-\theta}$. This means that the Lebesgue measure of $I_{n}$ goes to zero exponentially fast as $n \rightarrow \infty$. Thus, by the lemma of Borel-Cantelli, Lebesgue almost every point $x \in M$ belongs in only finitely many sets $I_{n}$. Clearly, any such point $x$ satisfies the conclusion of the lemma.

Let $\theta>0$ be the constant given by Lemma 6.1 and fix $\delta>0$ small enough so that $\sigma_{0}^{\theta}(1+\delta) \leq e^{-\lambda}$ for some $\lambda>0$. Let $x$ be any point satisfying the conclusion of the 
lemma. Then

$$
\prod_{j=0}^{n-1}\left\|D f\left(f^{j}(x)\right)^{-1}\right\| \leq \sigma_{0}^{\theta n}(1+\delta)^{(1-\theta) n} \leq e^{-\lambda n}
$$

for every large enough $n$. This implies that $x$ satisfies

$$
\limsup _{n \rightarrow+\infty} \frac{1}{n} \sum_{j=0}^{n-1} \log \left\|D f\left(f^{j}(x)\right)^{-1}\right\| \leq-\lambda .
$$

and since the conclusion of Lemma 6.1 holds Lebesgue almost everywhere we have that $f$ is a non-uniformly expanding map.

This shows that any sufficiently small neighborhood of $f$ in the $C^{2}$ topology constitutes a uniform family of non-uniformly expanding maps; cf. Definition 1.5.

\section{REFERENCES}

[1] J. F. Alves, SRB measures for non-hyperbolic systems with multidimensional expansion, Ann. Scient. Éc. Norm. Sup., $4^{e}$ série, 33 (2000), 1-32.

[2] _ Nonuniformly expanding dynamics: stability from a probabilistic viewpoint, Disc. and Cont. Dyn. Syst. 7, n.2 (2001), 363-375.

[3] J. F. Alves, C. Bonatti, M. Viana, SRB measures for partially hyperbolic systems whose central direction is mostly expanding, Invent. Math. 140 (2000), 351-398.

[4] J. F. Alves, S. Luzzatto, V. Pinheiro, Markov structures and decay of correlations for nonuniformly expanding dynamical systems, preprint CMUP 2002, arXiv:math.DS/0205191

[5] J. F. Alves, M. Viana, Statistical stability for robust classes of maps with non-uniform expansion, Ergod. Th. \& Dynam. Sys. 22 (2002), 1-32.

[6] V. Baladi, M. Viana, Strong stochastic stability and rate of mixing for unimodal maps, Ann. Scient. Éc. Norm. Sup., $4^{e}$ série, 29 (1996), 483-517.

[7] M. Benedicks, L. Carleson, On iterations of $1-a x^{2}$ on $(-1,1)$, Ann. Math. 122 (1985), 1-25.

[8] M. Benedicks, L.-S. Young, Absolutely continuous invariant measures and random perturbations for certain one-dimensional maps, Erg. Th. \& Dyn. Sys. 12 (1992), 13-37.

[9] R. Bowen, D. Ruelle, The ergodic theory of Axiom A flows, Invent. Math. 29 (1975), 181-202.

[10] - Equilibrium states and the ergodic theory of Axiom A diffeomorphisms, Lecture Notes in Mathematics 480 (1975), Springer.

[11] M. Jakobson, Absolutely continuous invariant measures for one-parameter families of onedimensional maps, Comm. Math. Phys. 81 (1981), 39-88.

[12] Yu. Kifer, Ergodic theory of random perturbations, Birkhäuser, Boston Basel, 1986.

[13] _ Random perturbations of dynamical systems, Birkhäuser, Boston Basel, 1988.

[14] D. Ruelle, A measure associated with Axiom A attractors, Amer. Jour. Math. 98 (1976), 619654.

[15] D. Ruelle, Differentiation of SRB states, Comm. Math. Phys. 187 (1997) n. 1, 227-241.

[16] Y. Sinai, Gibbs measures in ergodic theory, Russ. Math. Surv. 27, n. 4, (1972), 21-69.

[17] M. Viana, Multidimensional non-hyperbolic attractors, Publ. Math. IHES 85 (1997), 63-96.

[18] L.-S. Young, Stochastic stability of hyperbolic attractors, Erg. Th. \& Dyn. Sys. 6 (1986), 311-319.

[19] _ Recurrence times and rates of mixing, Israel J. Math. 110 (1999), 153-188.

CMup, Rua do Campo Alegre 687, 4169-007 Porto, Portugal

E-mail address: jfalves@fc.up.pt

$U R L:$ http://www.fc.up.pt/cmup/jfalves 